Vilca, H., Sosa, G., (2020) Etnogeometría aymara: propuesta de terminología matemática para la escuela rural de Perú. Revista Latinoamericana de Etnomatemática, 13(2), 66-86

DOI:10.22267/relatem.20132.61

Artículo recibido el 04 de mayo de 2020. Aceptado para publicación el 22 de junio de 2020

\title{
Etnogeometría aymara: propuesta de terminología matemática para la escuela rural de Perú
}

\section{Aymara ethnogeometry: proposal of mathematical terminology for the rural school of Perú}

\author{
Henry Mark Vilca Apaza \\ Fredy Sosa Gutierrez
}

\begin{abstract}
Resumen
El presente estudio es una aproximación a la etnogeometría aymara que tiene por objetivo la identificación de terminología matemática sobre geometría aymara, bajo el soporte epistemológico de la Etnomatemática y la interculturalidad, esperando que pueda tener una repercusión positiva en el aprendizaje y la identidad de los estudiantes de las escuelas rurales de Puno, donde existe problemas a causa de la interferencia lingüística. En el marco del método etnográfico, la información se obtuvo mediante visitas y entrevistas a los aymarahablantes de las comunidades de la provincia de Moho y El Collao de la región Puno - Perú, contrastada y complementada con fuente documental Vocabulario de la lengua aymara de 1612 y literatura especializada actual (libros y diccionarios). Los términos geométricos se identificaron por equivalencia y aproximación conceptual, y este proceso demostró que la lengua aymara cuenta con un rico bagaje léxico matemático propio y flexible para adaptarse a las exigencias científicas y pedagógicas actuales sea por creación de neologismos o préstamos de lenguas primas o extranjeras, en caso de vacíos. La terminología presentada en tablas fue escrita respetando las normas lingüísticas del aymara, y se espera sea estandarizada y socializada por las autoridades del Ministerio de Educación, y sirva de base a futuras discusiones e investigaciones.
\end{abstract}

Palabras clave: Terminología geométrica; Etnomatemática; Etnogeometría; Escuela Rural; Aymara.

\begin{abstract}
The present study is an approach to Aymara ethnogeometry that aims to identify mathematical terminology on Aymara geometry, under the epistemological support of Ethnomathematics and interculturality, hoping that it can have a positive impact on the learning and identity of students from rural schools in Puno, where there are problems due to linguistic interference. Within the framework of the ethnographic method, the information was obtained through visits and interviews with the Aymara speakers of the communities of the Moho and El Collao province of the Puno region - Peru, contrasted and supplemented with a documentary source Vocabulary of the Aymara language from 1612 and current specialized literature (books and dictionaries). The geometric terms were identified by equivalence and conceptual approximation, and this process showed that the Aymara language has a rich and flexible mathematical lexical background of its own to adapt to current scientific and pedagogical requirements, whether by creating neologisms or borrowing from raw or foreign languages, in case of gaps. The terminology presented in tables was written respecting the linguistic norms of Aymara, and it is expected that it will be standardized and socialized by the authorities of the Ministry of Education, and serve as the basis for future discussions and research.

\footnotetext{
* Doctor en Educación, Universidad Nacional del Altiplano, Puno, Perú, E-mail: hvilca@unap.edu.pe

** Doctor en Ciencias de la Educación, Universidad Nacional del Altiplano, Puno, Perú, E-mail: fredysosa@unap.edu.pe
} 
Key words: Geometric terminology; Ethnomathematics; Ethnogeometry; Rural School; Aymara.

\section{INTRODUCCIÓN}

\subsection{Interculturalidad, etnomatemática y terminología etnogeométrica del "otro"}

En un escenario rural y de habla aymara de Puno-Perú de fines del siglo XIX e inicios del XX, la educación oficial o "asimilacionista", como se la llamó, tuvo por propósito castellanizar al "indio" e incorporarlo al Estado-nación (López, 1988; Vilca, Yapuchura, Mamani \& Sardón, 2018). Los primeros maestros, de habla y didáctica castellana, se vieron impedidos de comunicarse, suscitándose problemas de aprendizaje, deterioro de identidad e interferencia lingüística en los alumnos, siendo la escuela "contraria al sentido ontológico que le otorga la comunidad aimara" (Bermejo-Paredes \& Maquera-Maquera, 2019, p.13). En el aspecto cognitivo, la comprensión de conceptos, en cultura e idioma ajenos, fue nula, siendo la matemática una de las áreas curriculares con mayores dificultades. Esta realidad motivó que Asunción Galindo en la Escuela Experimental de Ojherani-Puno, impulsara el método bilingüe consistente en la enseñanza inicial en lengua materna (aymara) y gradual transición al castellano. (Vilca et al., 2018). Esta y otras iniciativas sirvieron para que el Estado, en la década de los 70 del siglo XX, implemente la Educación Bilingüe (EB) y luego Educación Bilingüe Intercultural (EIB). Esta política educativa alterna y vigente para la escuela rural, que busca revalorar la lengua y cultura vernácula y facilitar el proceso de enseñanza-aprendizaje, en un área curricular como la matemática, requiere de profesores con un léxico especializado en aymara para acercarse al estudiante y pueda este comprender los conceptos de la matemática moderna. La lengua materna debe convertirse en un medio capaz de trasmitir los contenidos (López, 1988).

La interculturalidad, concebida como "diálogo armónico" (Perú), "reivindicación de derechos" (Bolivia y Ecuador) o "diálogo de poder" (Colombia), no se resume en una huera revaloración de la lengua y cultura o preservación de la sabiduría indígena, como un legado para las futuras generaciones (Guerra \& Guerra, 2017). Debe promover el auténtico diálogo de las culturas en todos los niveles de la vida, y ello implica, en primer lugar, en un mundo diverso y conflictivo a la vez, desarrollar el sentido humano de las personas para aprender a diferenciar sin disminuir (Reynoso, 2019); desde la complejidad (ecosofía, antropoética, 
Vilca, H., Sosa, G., (2020) Etnogeometría aymara: propuesta de terminología matemática para la escuela rural de Perú. Revista Latinoamericana de Etnomatemática, 13(2), 66-86

DOI:10.22267/relatem.20132.61

condición humana, etc.) (Guerra et al., 2017), fortalecer la humanidad del hombre como ente social y natural, el respeto a su dignidad, pues "la hominización es capital para la educación de la condición humana" (Morín, 1999, p.24). En segundo lugar, y como antesala al primero, implica fortalecer la intraculturalidad, empezando por redefinir la "cultura" de los pueblos originarios como un conjunto complejo de saberes y abandonar la concepción folclorista (cuentos, mitos y leyendas). Es necesario reconocer sus formas de gnosis y conocimientos sobre matemática, arquitectura, química, derecho, filosofía, literatura, música, medicina, ingeniería, religión, astronomía ancestral, sino científicos, como válidos. El reto no es sólo recuperar y conservar sino visibilizar y difundir esos conocimientos poniéndolos al servicio del bienestar de la vida y el hombre, desarrollando el campo intercientífico de la EIB dando valor cultural a la etnogeometría, la etnobiología, la etnomedicina (Guerra et al., 2017). De ahí que el estudio es una aproximación a la etnogeometría Aymara. La diversidad es posibilidad y no obstáculo para el desarrollo, como se pensó en el XX.

A la necesidad pedagógica y cultural, se suma la sed de justicia social y epistémica a los conocimientos de los pueblos indígenas que perviven a espaldas de la educación y ciencia oficial, debido al eurocentrismo y el academicismo, secundado en el Perú por la escuela asimilacionista-homogeneizante. Sin embargo, la epistemología crítica, la heterogeneidad, la pluriculturalidad, invitan a la apertura, a una mirada diferente al saber indígena, de razonar que hay tantas formas de conocer como civilizaciones hay en el planeta, de entender que las matemáticas, o cualquier área del saber, son diferentes en cada cultura y se expresan en contextos particulares (Ascher \& Ascher, 1986). La etnomatemática de D'Ambrosio (2005) es ese otro lente que se presenta como un programa de investigación de los diversos códigos de comportamiento en diferentes contextos culturales, socioeconómicos y naturales (ethno), para entender, explicar, conocer (mathema) formas, habilidades y técnicas (tica) de los diferentes pueblos, "no necesariamente ágrafos o no letrados" como dicen (Ascher et al. 1986). Aclara D'Ambrosio (2008) que la:

"Etnomatemática es un programa de investigación en historia y filosofía de las matemáticas, con implicaciones pedagógicas, que se sitúa en un marco muy amplio. Tu objetivo mayor es dar sentido a las formas de conocer y hacer diferentes culturas y 
reconocer cómo y por qué grupos de individuos... realizan sus prácticas matemáticas" (D’Ambrosio, 2008, p.1, traducido del portugués).

"La matemática es una de las construcciones humanas, cuyos modelos ideológicos... están anclados en la comprensión eurocéntrica de mundo y epistemológicamente estructurado por la ciencia moderna... -y estos han influido (agregado nuestro)- en la estética, la diversidad de estructuras, modelos de comportamiento, métodos y técnicas" (Ferreira \& Neves-Rogério, 2017, p.64).

La etnomatemática ofrece el marco referencial, la herramienta epistemológica, para demostrar que existen otras formas de matemática, de los pueblos que perviven al interior de los estados. Y no debería sorprendernos que dichas "habilidades prácticas no vengan acompañadas de un metalenguaje que las explique a nuestra entera satisfacción... nadie que no se haya entrenado en esos menesteres es capaz de dar cuenta verbalmente de la estructura de la lengua que habla" (Reynoso, 2019, p.137). Bajo este paradigma matemático, vale la acuñación de etno-geometría, entendida como la geometría no sólo de los pueblos originarios sino también de las barriadas, de la periferia, tribus, etc. Gerdes señala que "la etnogeometría es un conjunto de procedimientos metodológicos como forma de identificar elementos de pensamiento geométrico 'oculto' o 'congelado' en técnicas que tienen una larga historia” (2012, traducido del alemán y citado por Ferreira, et al., 2017, traducido del portugués, p. 66). Así, la etnogeometría se constituye en una herramienta teórica para realizar estudios contextualizados. Como dicen De la Hoz, Trujillo \& Tun (2017):

"En este contexto teórico, la etnomatemática es el análisis de conocimientos matemáticos desde un marco sociocultural y la etnogeometría provee un foro para la presentación y análisis de los patrones espaciales dentro del mismo marco sociocultural, que toma las formas inmateriales y abstractas de la geometría y las vuelve materiales y concretas al contextualizarlo dentro de una práctica y tradición cultural específica. Dentro del campo de la etnogeometría... tales objetos pueden revelar otra lógica de la construcción del pensamiento matemático y numérico" (De la Hoz et al., 2017, p.3). 
Vilca, H., Sosa, G., (2020) Etnogeometría aymara: propuesta de terminología matemática para la escuela rural de Perú. Revista Latinoamericana de Etnomatemática, 13(2), 66-86

DOI:10.22267/relatem.20132.61

Es necesario flexibilizar la marcha deductiva, el modelo formal, el lenguaje procedimental explícito, concebir que existe tantas geometrías como pueblos hay, hablar de geometrías en plural y ya no de geometría en singular, descolonizarnos de que surgió únicamente en el antiguo Egipto (3100 a 332 a.C.), y que es la única válida por ser ciencia del razonamiento lógico (siglo VI a.C. con Thales) y deductiva con Euclides. Reynoso (2019) precisa:

"Las pocas ocasiones en las que en Occidente se habló de geometría siempre se trató de la geometría euclidiana plana, platónica y lineal... tendremos ocasión desde el inicio de abordar las geometrías en plural, dando cabida a las muy diversas geometrías de la fractalidad compleja e hipercompleja, de los volúmenes paradójicos, de los embaldosados no periódicos, de las transformaciones recursivas y no lineales, de las propiedades emergentes, de las trayectorias multifurcadas y de las superficies curvas esféricas, parabólicas e hiperbólicas a las que aquí se darán preeminencia por cuanto operan como las geometrías alternativas y descentradas a la luz de las cuales... las prácticas que consideramos más extrañas, inexplicables y heterodoxas adquieren sentido" (Reynoso, 2019, p. 6).

En ese marco, fue objetivo del presente identificar y sistematizar la terminología matemática de la geometría Aymara, a fin de ser incorporada en el proceso de enseñanza aprendizaje en de escuelas rurales en pro del aprendizaje y la identidad cultural, en el marco de la EIB, esperando pueda generar discusiones e investigaciones futuras.

\section{METODOLOGÍA}

El grupo cultural Aymara comprende a los países de Bolivia, Perú, Chile (CerrónPalomino, 2000) y Argentina. En Perú, 548311 personas se auto identificaron como tal, según el último censo de 2017, que esencialmente están en la región Puno, al sur de Perú, que fue la zona de intervención. El estudio se valió del método etnográfico para acceder al conocimiento de las comunidades, y del método descriptivo - analítico para realizar un análisis contrastivo de la información obtenida con fuentes bibliográficas, para mayor sostenibilidad. La etnometodología de Gerdes empleada por Ferreira et al. (2017), sugiere primero aprender la técnica, medio y forma del quehacer matemático, en nuestro caso no fue necesario puesto que se limitó a la observación y al recojo de términos por entrevista, y no se orientó a un estudio antropológico ni interpretativo. 
La etapa de recolección de información (2016 al 2020) empezó con la elaboración de la guía de entrevista con preguntas abiertas que buscaron el significado de cada palabra como ¿kunasa jumataki jullisaltaxa? (¿Qué es geometría para ti?) ¿Kuna jullisaltanaxsa uñt'axptaxa? (¿Qué figuras conocen?) o ¿Kamsañasa mujinaxa? ¿Cómo se dice triángulo en aymara?), que se aplicaron, en diferentes visitas al domicilio de los participantes mayores de 60 años de las comunidades de la provincia de Moho (codificado con A) y de El Collao (codificado con B). Los términos se identificaron por equivalencia $\mathrm{y}$ aproximación conceptual. Las respuestas fueron registradas en la lista de términos geométricos Castellano-Aymara, previamente estructurado. Esta etapa fue auxiliada de la observación por visita guiada a diversos lugares (viviendas, sembríos, restos arqueológicos, etc.) y exhibición de prendas (gorra y manta) que evidencian representaciones geométricas. La información recogida fue corregida, ratificada y traducida por el participante Miguel Mamani que tuvo el rol de traductor por ser bilingüe castellano-aymara y trabajador del sector educación. Los términos no encontrados en la voz de los hablantes, fueron complementados y consolidados con el análisis documental, es decir con revisión del vocabulario de la lengua Aymara de 1612 de Ludovico Bertonio (1993), fuente colonial y primaria sobre el tema, escrita a inicios de la conquista española $\mathrm{y}$, reforzados y contrastados con diccionarios español/aymara escritos por educadores y lingüistas Aymaras, y libros actuales. Una segunda etapa, previa a la presentación tablas, fue la traducción, revisión, y validación fonológica y morfosintáctica de los términos por un experto en lingüística Aymara (coautor) y de acuerdo con la Resolución Ministerial $\mathrm{N}^{\mathrm{o}}$ 1218-85-ED del Ministerio de Educación Perú, que establece las grafías para la lengua aymara.

\section{RESULTADOS}

\subsection{Aymara Saltatuqita: geometría aymara}

La terminología geométrica aymara está en las actividades que realiza, en los objetos que crea (textilería, alfarería, agricultura, etc.) y en el lenguaje oral. El término que engloba este área del saber (geometría) es salta (Deza, 1989) o saltatuqita (según A y B) de salta $=$ gráficos geométricos y tuqita = acerca de, validado por el Ministerio de Educación del Perú 
Vilca, H., Sosa, G., (2020) Etnogeometría aymara: propuesta de terminología matemática para la escuela rural de Perú. Revista Latinoamericana de Etnomatemática, 13(2), 66-86

DOI:10.22267/relatem.20132.61

(Minedu, en adelante); sin embargo, Miguel Mamani (comunicación personal, setiembre 20, 2018) precisa que más usual es pacha tupuña, de pacha = espacio y tupuña = medida, como la etimología griega "medida de la tierra". Sus elementos (yänanaka en aymara) más notorios son: recta equivale a chiqaki (recta, según A y B) y siq’i (línea recta, para Bertonio, 1612); punto, a ch'aqa (gota); recta paralela, a jikiwischiqaki o kiksachiqaki (según el Minedu, 2002); recta secante, a chakanata (cruzado) o chiqaki; recta perpendicular, a chakata chiqaki o sayxatatchiqaki (Minedu, 2002); semirecta, a chikchiqaki; y segmento, a chimputchiqaki. El término “ángulo" equivale a k’uchu, de rincón, sostenido por Layme (2004) y Minedu (2002 y 1905), que según este último equivaldría además a q’ichu. Este dato sirve para reconstruir la familia terminológica de ángulo (Tabla 1). Por ejemplo, transportador es a k’uchu tupuña, de k’uchu = ángulo y tupuña $=$ medir.

\begin{tabular}{|c|c|c|}
\hline \multirow[t]{2}{*}{ Castellano } & \multicolumn{2}{|c|}{ Aymara } \\
\hline & Equivalente & Glosa \\
\hline Elementos & Yänanaka & Cosas, objetos \\
\hline Geometría & Salta, saltatuqita, pacha tupuña & Medición del espacio \\
\hline Recta & Chiqaki, siq'i & Que va recto o línea recta \\
\hline Semirrecta & Chikchiqaki & Parte de la recta \\
\hline Segmento & Chimputchiqaki, chimputasich'i & Entre dos puntos \\
\hline Punto & Ch'aqa & Punto, gota \\
\hline Rectas paralelas & Jikiwischiqakinaka, kiksa chiqakinaka & Rectas que van juntas \\
\hline Rectas secantes & Chakanata, chiqakinaka & Rectas cruzadas (como la Cruz del sur) \\
\hline Rectas perpendiculares & Sayxatatchiqakinaka, Chakata chiqakinaka & Que se posiciona recto \\
\hline Ángulo & K'uchu, q'ichu & Rincón interno, esquina \\
\hline Ángulo agudo & K’ullku k’uchu & Rincón angosto \\
\hline Ángulo recto & Chiqa k'uchu, chiqapa k’uchu & Rincón recto \\
\hline Ángulo obtuso & Jist'arata k'uchu & Rincón muy abierto \\
\hline Ángulo llano & Jant'akuta k'uchu & Muy estirado \\
\hline Transportador & K’uchu tupuña & Medir las esquinas \\
\hline
\end{tabular}

Tabla 1. Términos sobre elementos de la geometría.

Fuente: Lista de términos castellano-aymara.

\subsection{Jullisaltanaka: figuras geométricas}

La textilería es obviamente el mayor registro de la geometría Aymara, es la evidencia del quehacer y pensar geométrico. No es mera indumentaria, es la memoria viva de sus conocimientos, terminología y simbología. En las llijlas (mantas), wach us (gorras) se advierte figuras fitomórficas de qhantuta (flor de la cantuta), panqara (flor, como en la Figura 2); astros como inti (sol), phaxsi (luna); figuras zoomorfas de jamp'atu (sapo), 
kunturi (cóndor), asiru (culebra), philpintu (mariposa); figuras antropomorfas; simbología como la espiral signo del pensamiento cosmológico; etc., con significado e interpretación de acuerdo al color y la presencia de otras figuras, como el cuadrado, triángulo, rombo, rectángulo, rectas, polígonos, como se aprecia en la Figura 1, siendo fuente de rica terminología.
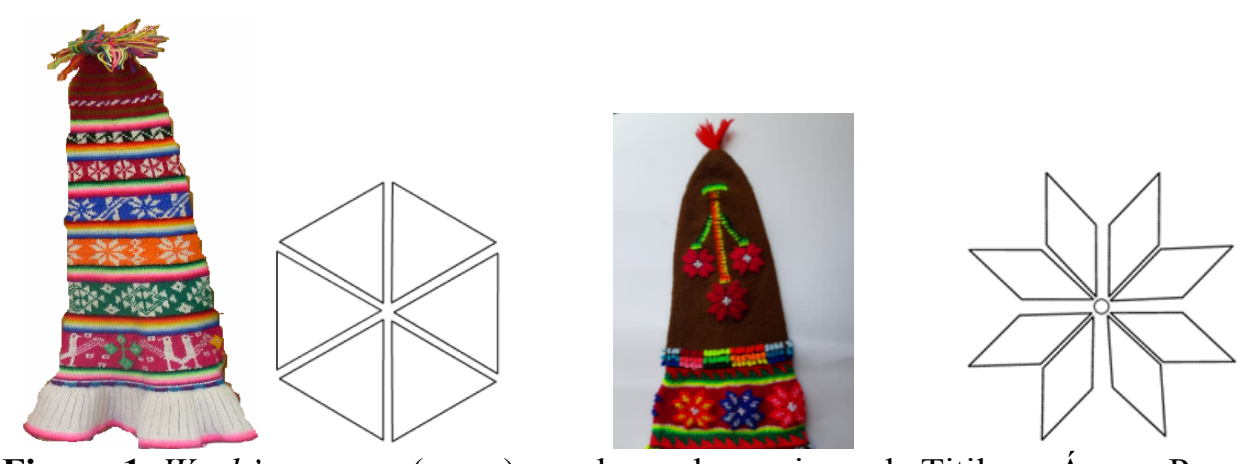

Figura 1. Wach'u aymara (gorra) usada por las mujeres de Titilaca, Ácora, Puno.

Fuente: Archivo de Henry Vilca-Apaza, tomada en 2020

La agricultura y las tecnologías agrícolas aymaras son desarrolladas bajo conceptos geométricos, por ejemplo la qutaña (laguna artificial), waru waru (camellones) patapatas (andenes) (Figura 5). Desde la cima de un cerro se observa que los sembríos de las comunidades están distribuidos geométricamente simbolizando diferentes productos. Ver Figura 2. Las tejedoras de la parcialidad de Sikupumawqa, Moho, indican que:

"los triángulos rectángulos significan chacras de habas y cebadas; los pentágonos, chacras de quinua y cañihua; el cuadrado o rectángulo, chacra de papa; el triángulo isósceles, chacra de haba. Los pequeños rombos y la pequeña circunferencia dentro de estas figuras geométricas significarían que son chacras a punto de ser cosechadas o producto base y de consumo universal, respectivamente; la ausencia de éstas, terreno por preparar" (Arias, 2005, p. 41). 
Vilca, H., Sosa, G., (2020) Etnogeometría aymara: propuesta de terminología matemática para la escuela rural de Perú. Revista Latinoamericana de Etnomatemática, 13(2), 66-86

DOI:10.22267/relatem.20132.61

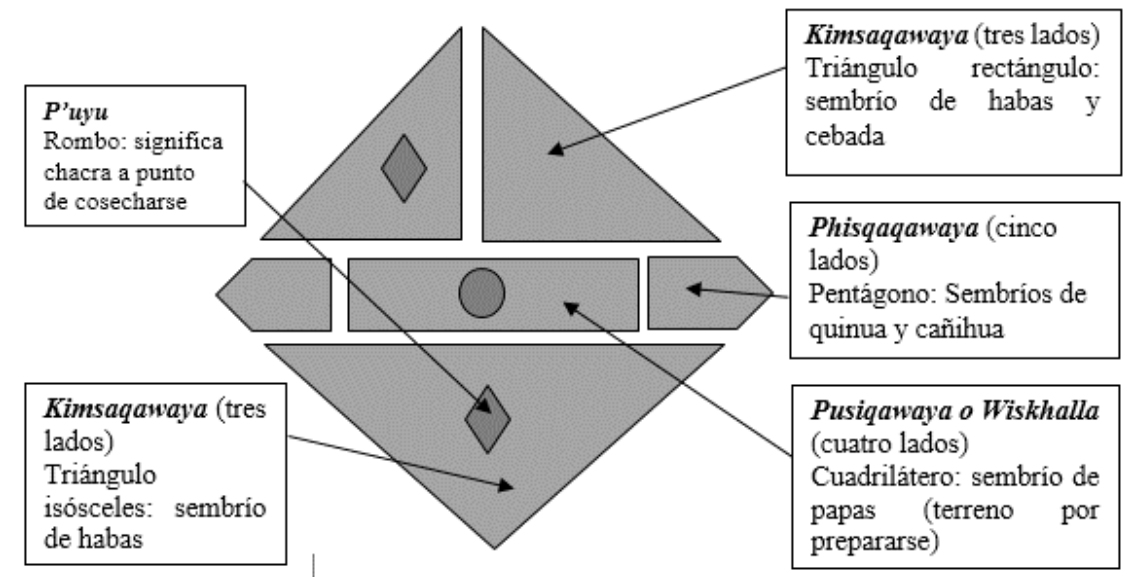

Figura 2. Mayt'u y simbología en la agricultura aymara-lupaca (Moho, Puno).

Fuente: Recreación con base en Arias (2005)

Con base a la entrevista y la literatura peruano-boliviana, se presenta la terminología sobre figura geométrica en aymara en la Tabla 2. El término "figura geométrica" se compone de “figura", que según Bertonio (1612) es ulljnaqa (en desuso) y julli según Minedu (1905), y de salta que equivale a "geometría". En consecuencia "figura geométrica" equivale a jullisalta, y en plural, jullisaltanaka, que resulta de adicionar el sufijo pluralizador -naka.

\begin{tabular}{ll}
\hline & Castellano \\
\hline Figura & Julli, uñta \\
\hline Figura geométrica & Jullisalta, Salta, salta tupuña \\
\hline Figura plana & Pallalla salta, p'axla salta \\
\hline Círculo & Muyu \\
\hline Cuadrado & Pusi k'uchu, pusi k'uchuni kajuna, sinilla \\
\hline Triángulo & kimsa k'uchuni, mujina, mujiña \\
\hline Rectángulo & sayt'u, wiskhalla kajuna \\
\hline Circunferencia & Muyuthiya \\
\hline
\end{tabular}

Tabla 2. JULLISALTANAKA (Figuras geométricas).

Fuente: Lista de términos castellano-aymara.

Círculo (тиуи). Esta forma se observa en las qutañas, tecnología agrícola que consiste en lagunillas artificiales abastecidas por agua de río e intercomunicadas mediante canales que sirven de bebedero de animales, aclimatador natural y humedal para vegetación y agricultura. Hay dos formas: muyu qutaña (lagunilla circular) y sayt'u qutaña (lag. rectangular). Por otro lado, el "Collar de Ilave" (ver Figura 3), que data de 4500 a.C., hallada a $15 \mathrm{~km}$ del distrito de Ilave, provincia de El Collao-Puno, refleja formas circulares (Fernández, 2008). El círculo, figura plana formada por la circunferencia y su interior, equivale, al igual que en quechua (Gonzales, 1608), a mиyu (según A y B). Ver Tabla 2. 
También está muyunkutu y sinku, como dialectos. Sus elementos son muytatupu (perímetro), muyjalja (diámetro), muytiri (radio), waru waruru (longitud, según Layme, 2004) у pampatupu (área). Circunferencia equivale a muyuthiya (Minedu, 2002 y Layme, 2004). Ver Tabla 3.
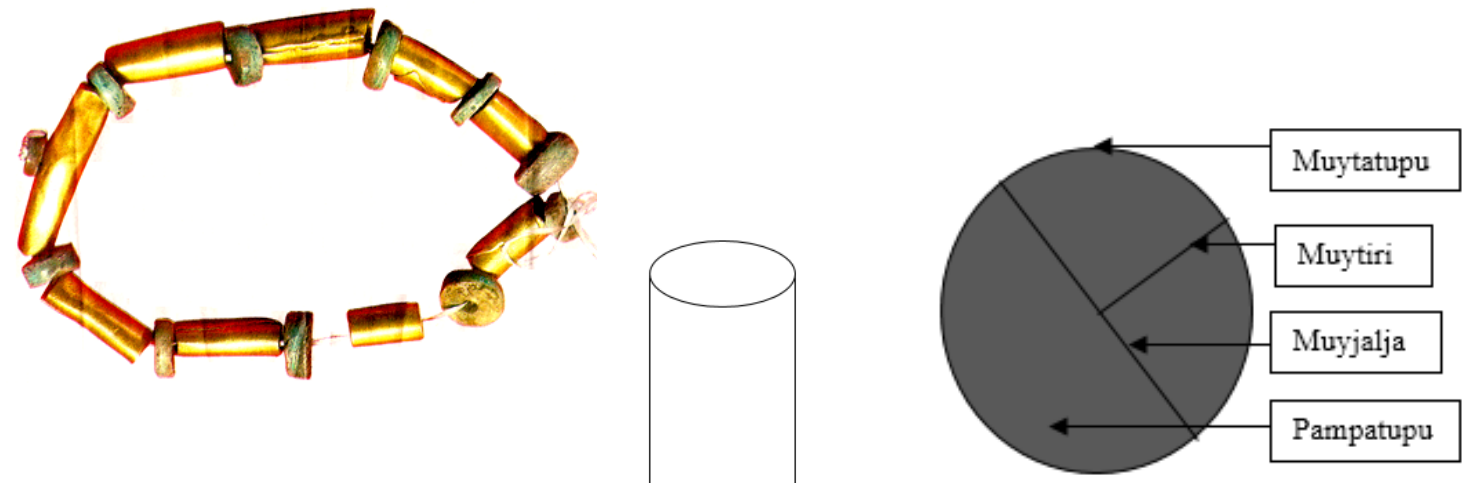

Figura 3. El Collar de Ilave (I), reproducción del cilindro (C) y del círculo (MUYU) y partes (D) Fuente: Fernández (2008) (I). Reproducción libre (C y D)

\begin{tabular}{lll} 
& \multicolumn{2}{c}{ Cysmara } \\
\cline { 2 - 3 } Castellano & Equivalente & Glosa \\
\hline Perímetro & Muytatupu & Longitud circular \\
\hline Radio & Muytiri & Que da vueltas \\
\hline Diámetro & Muyjalja, chikjaljkira & Corta por la mitad \\
\hline Longitud & Waru waruru & Distancia \\
\hline Área & Pampatupu & Espacio que ocupa \\
\hline Vértice & Ari & Punta filuda \\
\hline Lado & Thiya, qawaya & Al costado \\
\hline Ángulo & K'uchu & Rincón (interno) \\
\hline Base & Ch'ina & Base \\
\hline Altura & Tansa & Altura \\
\hline Ancho & Irwaqa, qhanqha & Ancho \\
\hline Largo & Wiskhalla, waru, whiskhalla sayt'u & Largo, alargado \\
\hline Unidades de medida & Tupuñanaka & Medidas \\
\hline
\end{tabular}

Tabla 3. Elementos de las figuras geométricas.

Fuente: Lista de términos castellano-aymara.

Cuadrado (pusi k'uchu).- Este polígono de cuatro lados iguales se puede encontrar en putukus, chullpas, tejidos, cerámicas, etc., cuya denominación en aymara es: kajuna, pusi iskina y pusi k’uchu. El primero es una aymarización de 'cajón'; el segundo, pusi iskina, contiene la palabra iskina del castellano, por lo que ambos son inválidos. La opción 'pusi 
Vilca, H., Sosa, G., (2020) Etnogeometría aymara: propuesta de terminología matemática para la escuela rural de Perú. Revista Latinoamericana de Etnomatemática, 13(2), 66-86

DOI:10.22267/relatem.20132.61

k’uchu (Layme, 2004, Deza, 1989 y según A y B), que vale decir pusi = cuatro, k'uchu= rincón, es la más adecuada, traducido como "cuatro ángulos". Los elementos identificados son ch'ina, (base), thiya (lado, y no es qawaya que significa costado), ari (vértice) y k’uchu (ángulo).

Triángulo (mujiña).- Este polígono, muy visual en la textilería (ver Figura 1), tiene la siguiente opciones: kimsa iskina (según A y B), kimsa k'uchuni, mujina (Layme, 2004) y mujiña (Minedu, 2002). Nótese que en el primero, iskina es aymarización de Esquina, y el segundo es la forma descriptiva (que tiene tres rincones), inválidas por esas razones. La opción válida es mujiña y mujina, cuyos elementos son: vértice igual a ari; lado, qawaya (según A y B) y thiya (Layme, 2004); base, ch’ina ("base del objeto"); altura, tansa (Minedu, 1905 y Bertonio, 1612) y sayt’u (Bertonio, 1612). En la Tabla 4, también se presenta terminología sobre los tipos de triángulo, producto de neologismos en base a proximidad conceptual. Por sus lados, el triángulo equilátero, isósceles y escaleno en aymara equivale a kikpqawaymujiña, mayjqawaymujiña y sayt'umujiña, respectivamente; mientras que según la medida de sus ángulos, sólo se identificó para rectángulo que es wiskhalla. Ver Tabla 4.

\begin{tabular}{lll}
\hline \multirow{2}{*}{ Por su lado } & \multicolumn{1}{c}{ Castellano } & \multicolumn{1}{c}{ Aymara } \\
\cline { 2 - 3 } & Triángulo equilátero. (tres lados iguales) & Kikpqawaymujiña, kikqawaya mujiña \\
\cline { 2 - 3 } & Triángulo isósceles. (dos lados iguales). & Mayjqawaymujiña \\
\cline { 2 - 3 } & Triángulo escaleno. (tres lados distintos). & Sayt'umujiña \\
\hline \multirow{2}{*}{$\begin{array}{l}\text { Por su } \\
\text { medida }\end{array}$} & Acutángulo (tres ángulos internos agudos) & $\ldots$ \\
\cline { 2 - 3 } & Rectángulo (ángulo de $90^{\circ}$ ) & Wiskhalla \\
\cline { 2 - 3 } & Obtusángulo ( mayor a $\left.90^{\circ}\right)$ & Jist'arata k'uchu \\
\hline
\end{tabular}

Tabla 4. Terminología - tipos de triángulo.

Fuente: Lista de términos castellano-aymara.

Rectángulo (sayt'u).- Esta figura está presente en la Qhunaña, instrumento doméstico de piedra para triturar granos andinos como quinua y cañihua y obtener harina $\left(j a k^{\prime} u\right)$ (ver Figura 4). Se observa también en la Sayt'u qutaña y, en los camellones (Waru Waru), tecnología agrícola iniciada por la cultura Pukara hacia 800 a.C. (Palao, 2005), que consiste en elevar el suelo por encima del nivel de la superficie natural del terreno, logrando plataformas de cultivo intercaladas por canales llenas de agua con el propósito de disminuir la acción devastadora de las heladas y contrarrestar las inundaciones. Esta forma 
geométrica tiene las siguientes equivalencias: wiskhalla kajuna, sayt'u, pusi iskina wiskhalla. Las palabras pusi iskina wiskhalla (pusi = cuatro, iskina = esquina y whiskhalla = largo) y wiskhalla kajuna (whiskhalla = largo y kajuna = cajón) quedan descartadas por ser descriptivas y aymarizadas. La opción más razonable es la de Layme (2004), sayt’u, pues refiere la forma alargada.
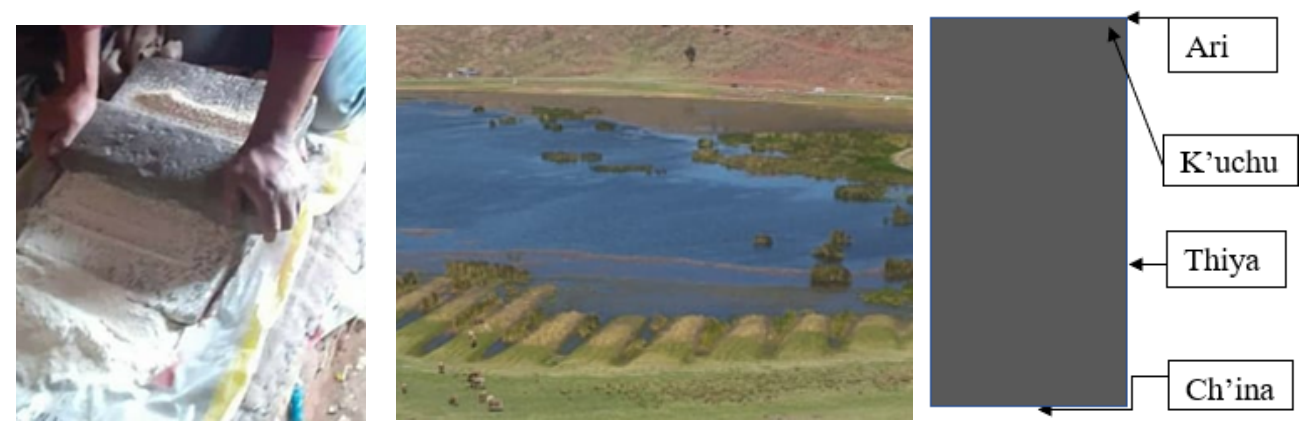

Figura 4. Qhunaña (molino) (I), Waru Waru o Camellones (C) y recreación de rectángulo (SAYT'U) (D).

Fuente: Fotos tomadas por Henry Vilca-Apaza, 2019 (I y C).

Los Polígonos.- Un polígono es la figura que tiene varios lados, cuya equivalencia en aymara por aproximación conceptual es waljaqawaya (según Ay B), etimológicamente $w a l j a=$ varios y qawaya $=$ lado, asimilado por Minedu. En plural se pronuncia como waljaqawayanaka, es decir se agrega - naka, sufijo flexivo nominal de número (CerrónPalomino, 2000). La estructura léxica de la familia de polígonos, (ver la Tabla 5), presenta el fenómeno lingüístico llamado "caída vocálica", característico del aymara. En Eneágono que es llätunkqawaya (llätunka = diez y qawaya = lado) se observa la caída de la vocal "a".

\begin{tabular}{|c|c|c|}
\hline \multirow[t]{2}{*}{ Castellano } & \multicolumn{2}{|c|}{ Aymara } \\
\hline & Equivalente & Glosa \\
\hline Polígono & Waljaqawaya & Varios lados \\
\hline Triángulo & Kimsaqawaya o Mujiña & Tres (kimsa) lados (qawaya) \\
\hline Cuadrilátero & Pusiqawaya & Cuatro lados \\
\hline Pentágono & Phisqaqawaya & Cinco lados \\
\hline Hexágono & Suxtaqawaya & Seis lados \\
\hline Heptágono & Paqallqqawaya & Siete lados \\
\hline Octógono & Kimsaqallqqawaya & Ocho lados \\
\hline Eneágono & Llätunkqawaya & Nueve lados \\
\hline Decágono & Tunkaqawaya & Diez lados \\
\hline Dodecágono & Tunkapäqawaya & Doce lados \\
\hline Icoságono & Pätunkaqawaya & Veinte lados \\
\hline
\end{tabular}

Tabla 5. WALJAQAWAYA: Polígonos y sus tipos.

Fuente: Lista de términos castellano-aymara. 
Vilca, H., Sosa, G., (2020) Etnogeometría aymara: propuesta de terminología matemática para la escuela rural de Perú. Revista Latinoamericana de Etnomatemática, 13(2), 66-86

DOI:10.22267/relatem.20132.61

Los cuadriláteros (pusiqawayanaka) se clasifican en paralelogramos (jikiwisqawayanaka), trapecios (qhapananaka) y trapezoides (sin equivalente) (ver Tabla 6). El paralelogramo, en aymara es jikiwisqawaya (jikiwisa = paralelo y qawaya $=$ lado). El término rombo equivale a p’uyu у ch'uyu (según A y Layme, 2004); trapecio a qhapana en singular y qhapananaka en plural. Un vacío terminológico para romboide, trapecio escaleno e isósceles.

\begin{tabular}{ll}
\hline \multicolumn{1}{c}{ Castellano } & Aymara \\
\hline Paralelogramo & Jikiwisqawaya \\
\hline Romboide & $\ldots$ \\
\hline Rombo & P'uyu, ch'uyu \\
\hline Rectángulo & Wiskhalla, sayt'u \\
\hline Cuadrado & Pusi k'uchu \\
\hline Trapecio & Qhapana \\
\hline Trapecio escaleno & $\ldots$ \\
\hline Trapecio isósceles & $\ldots$ \\
\hline Trapecio rectángulo & Sayt'u qhapana \\
\hline
\end{tabular}

Tabla 6. Terminología - clases de paralelogramos y trapecios.

Fuente: Lista de términos castellano-aymara.

El término perímetro, común en el control de terrenos empleando medidas arbitrarias como chilqi y kayu que son motivo de otro estudio, en aymara es muyta tupu (Minedu, 2002) y sus elementos thiya (lado), irwaqa o qhanqha (ancho, según A y B y Bertonio, 1612), waru (Minedu, 2002) o whiskhalla sayt'u (largo, Bertonio, 1612) y tupuñanaka (unidades de medida) (ver Tabla 3). Plano cartesiano es chakata chiqakinaka pampa, refonologización y propuesta del Minedu (2002). El eje de las abscisas es winkutaru kuchuri (echado) y el de las ordenadas, sayataru kuchuri. El término "plano" equivale a Pampa (según A y Layme, 2004), p’alta (según Deza, 1989) y p'allalla (según A y Minedu, 2002). Por otro lado, par ordenado se traduce como siqichata payata (Minedu, 2002), que traducido es payata = de dos y siqichata $=$ ordenado. Ver Tabla 7.

\begin{tabular}{lll}
\hline \multirow{2}{*}{ Castellano } & \multicolumn{1}{c}{ Equivalente } & Glosa \\
\cline { 2 - 3 } & \multicolumn{1}{c}{ Pampa, p'alta, p'allalla } & Plano \\
\hline Plano & Chakata chiqakinaka pampa & Plano recto cruzado \\
\hline Plano cartesiano & Sayataru kuchuri & Parado \\
\hline Eje de las ordenadas & Winkutaru kuchuri & Echado \\
\hline Eje de las abscisas & Siqichata payata & De a dos \\
\hline Par ordenado &
\end{tabular}

Tabla 7. Términos sobre el plano cartesiano. 
Fuente: Lista de términos castellano-aymara.

\subsection{Tikanaka: cuerpos geométricos}

Los Uro-Pukina, que viven en las islas flotantes del lago Titicaca, fue un grupo amazónico, que remontando los ríos llegaron a la cordillera de Perú, para desplazarse por el altiplano hasta llegar al lago, asentándose a las orillas de los ríos en viviendas construidas con champas, llamadas putukus, una arquitectura asimilada por los aymaras, sus conquistadores, que sin duda es la evidencia del temprano desarrollo conceptual geométrico en el altiplano. La denominación de "cuerpo geométrico" en aymara es jullitunu según Minedu (2002), y según (Layme 2004), tika, optándose por esta última, pues julli es figura.

\begin{tabular}{lll}
\hline \multirow{2}{*}{$\begin{array}{l}\text { Cuerpo } \\
\text { básico }\end{array}$} & \multicolumn{1}{c}{ Castellano } & \multicolumn{1}{c}{ Aymara } \\
\cline { 2 - 3 } & Esfera & Tika, jullitunu \\
\cline { 2 - 3 } & Cubo & Muruq'u \\
\cline { 2 - 3 } & Cilindro & Suxta ajanu, kajuna \\
\cline { 2 - 3 } & Cono & Sayt'umuyu, t'uyu \\
\hline \multirow{2}{*}{ Elementos } & Superficie lateral curva & Kalicha, t'ajta ariru tukuyata \\
\cline { 2 - 3 } & Base & Pampa, muyta \\
\cline { 2 - 3 } & Altura & Ch'ina \\
\cline { 2 - 3 } & Vértice & Tansa \\
\cline { 2 - 3 } & Superficie lateral curva & Ari \\
\hline
\end{tabular}

Tabla 8. TIKANAKA: Cuerpos geométricos básicos.

Fuente: Lista de términos castellano-aymara.

Esfera (muruq'u).- Un objeto fabricado por aymaras que exhibe este geométrico es el muruq'u qala (piedra redonda), parte del batán (k'iyaña en aymara), un instrumento doméstico para moler. En lengua aymara "esfera” es muruq’u según versión de A y B, y Deza (1989), en tanto en quechua es rumpu (Gonzales, 1608). Para Bertonio (1612) es mulluq'u.

Cubo (suxta ajanu).- Como se ve en la tabla 8, la literatura proporciona dos posibilidades para este cuerpo: Suxta ajanu y kajuna. La última posibilidad (kajuna) queda descartada porque es una refonologización ociosa de “cajón”. El primero, suxta ajanu (según A y B y Layme, 2004) muestra mejor aproximación conceptual y se traduce como "seis caras".

Cilindro (sayt'umuуu, tuyu). - Formas cilíndricas se observan en las chullpas del altiplano peruano, construcciones arquitectónicas aymaras que sirvieron de monumento mortuorio, de 1.5 a 8 metros de altura, construidas con el fin de rendir culto al curaca fallecido 
Vilca, H., Sosa, G., (2020) Etnogeometría aymara: propuesta de terminología matemática para la escuela rural de Perú. Revista Latinoamericana de Etnomatemática, 13(2), 66-86

DOI:10.22267/relatem.20132.61

(Kesseli \& Pärssinen, 2005). En Molloco, ubicado en la comunidad del mismo nombre, distrito de Ácora, provincia y departamento de Puno, además de cuadradas se observa una chullpa cilíndrica de planta circular (ver Figura 5), cuyo proceso constructivo debió obligar a acuñar términos geométricos. Este tipo de cuerpo equivale a sayt'umuyu (círculo alargado) y t'uyu que tiene el respaldo del Minedu. Los elementos del cilindro son: pampa (superficie lateral curva), ch’ina (base) y tansa (altura) según Bertonio, (ver Tabla 8).
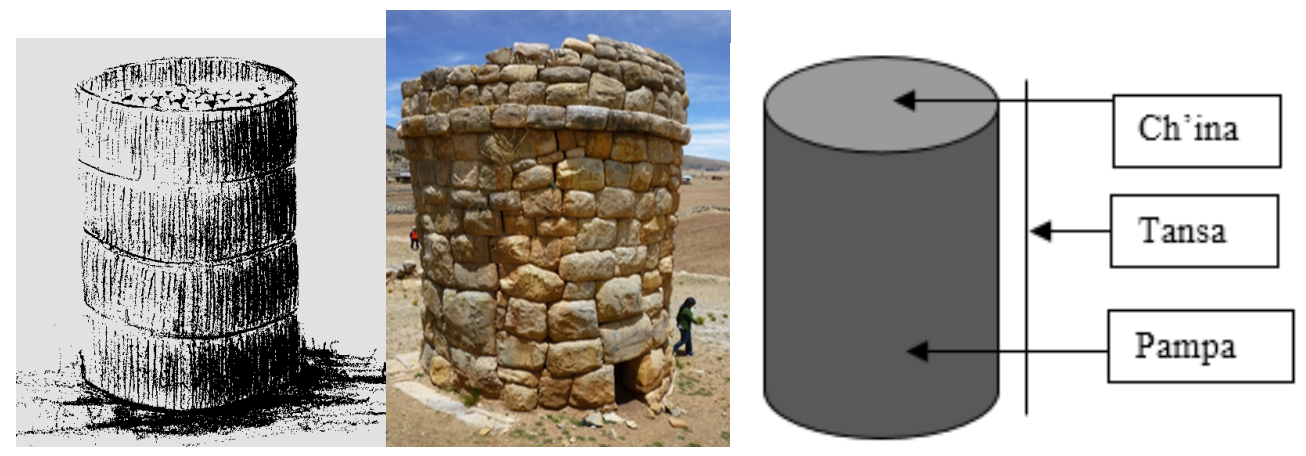

Figura 5. Sixi, depósito cilíndrico para produtos (I), Chullpa cilíndrica de base circular de Molloco (C). Reproducción del cilindro y sus elementos (SAT'UMUYU) (D).

Fuente: Dibujo libre (I), foto tomada por Henry Vilca-Apaza, 2007 (C).

Cono (kalicha).- Al igual que en Colombia, donde los arhuacos en la construcción de su vivienda tradicional establecen conceptos geométricos y de relaciones matemáticas como las proporciones (De la Hoz, et al., 2017), en el altiplano peruano las viviendas reflejan geometría. En el centro arqueológico de Tanqa Tanqa (conocido como Pukara) ubicado a $172 \mathrm{~km}$ al sur de Puno, en el distrito de Kelluyo, provincia de Chucuito, departamento de Puno, además de chullpas prismáticas se observan otras cónicas de planta circular (ver Figura 6) cuya construcción es iterativa con bloques cúbicos de piedra. Este cuerpo también se observa en el Chuku o gorra aymara de color amarillo (Museo Municipalidad de Juli, Puno) perteneciente al reino aymara Lupaca asentado a orillas del Titikaka (1100 a 1450 d.C.) y en las chujllas (casas cónicas de totora). Este cuerpo geométrico equivale a kalicha o t'ajta ariru tukuyata equivalente a "Cono", siendo válido el primero pues el segundo es la forma descriptiva (cuerpo plano que termina en punta). El cono tiene una base circular (ch'ina), una superficie lateral curva (pampa), y altura (tansa). 

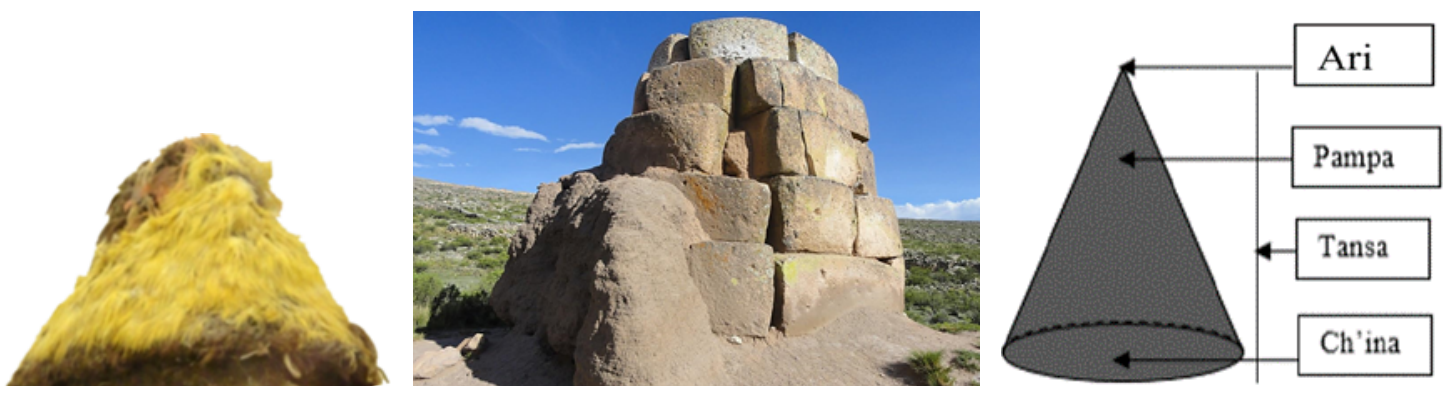

Figura 6. Chuku lupaca (I), Kalicha o chullpa cónica (C) y representación gráfica (KALICHA) (D).

Fuente: Foto tomada por Henry Vilca-Apaza, 2019 (I) y 2015 (C).

\begin{tabular}{|c|c|c|c|}
\hline & \multirow[t]{2}{*}{ Castellano } & \multicolumn{2}{|c|}{ Aymara } \\
\hline & & Equivalente & Glosa \\
\hline \multirow[t]{6}{*}{ Regulares } & Poliedro & Waljajanu & Varias caras \\
\hline & Tetraedro & Pusiajanu & Cuatro caras \\
\hline & Exaedro o cubo & Suxtäjanu & Seis caras \\
\hline & Octaedro & Kimsaqallqajanu & Ocho caras \\
\hline & Dodecaedro & Tunkapayanajanu & Doce caras \\
\hline & Icosaedro & pätunkäjanu & Veinte caras \\
\hline \multirow[t]{2}{*}{ Irregulares } & Prisma & Pirwa & $\ldots$ \\
\hline & Pirámide & Wamp'ara, putuku & \\
\hline
\end{tabular}

Tabla 9: WALJAJANU: Poliedros.

Fuente: Lista de términos castellano-aymara.

Poliedros.- Los cuerpos geométricos se dividen en poliedros y cuerpos redondos. Poliedro, por equivalencia conceptual, es waljajanu en aymara, cuya etimología es walja = varios y ajanu $=$ cara, igual a "varias caras". Las denominaciones en aymara de los poliedros regulares e irregulares se presentan en la Tabla 9, por ejemplo, Icosaedro es pätunkäjanu, de $p \ddot{a t u n k} a=$ veinte y ajanu $=$ cara .

Pirámide (wamp'ara).- Este poliedro irregular que tiene como base un polígono y cuyas caras laterales son triángulos con un vértice común opuesto a la base, en aymara es putuku (por la forma parecida) o wamp'ara (según $\mathrm{MM}$ ), término base para reconstruir la terminología acerca de los tipos de pirámide. Por ejemplo, "pirámide triangular" equivale a mujina wamp'ara (mujina = triángulo y wamp'ara = pirámide). Sus elementos son k'achi (arista), ari (vértice), qawaya k'achi (arista lateral), ch'ina k'achi (arista base), ch'ina (base), ајапи (cara) y thiya (lado). (ver Tabla 10)

\begin{tabular}{|c|c|}
\hline & Aymara \\
\hline Vértice & Ari \\
\hline Arista & K'achi \\
\hline Arista lateral & Qawaya k'achi \\
\hline
\end{tabular}


Vilca, H., Sosa, G., (2020) Etnogeometría aymara: propuesta de terminología matemática para la escuela rural de Perú. Revista Latinoamericana de Etnomatemática, 13(2), 66-86

DOI:10.22267/relatem.20132.61

\begin{tabular}{ll}
\hline Arista básica & Ch'ina k'achi \\
\hline Base & Ch'ina \\
\hline Cara & Ajanu \\
\hline Lado & Thiya \\
\hline Área, superficie & Pampa, pampa tupu \\
\hline Espacio & Chhaqa \\
\hline
\end{tabular}

Tabla 10. Elementos del poliedro.

Fuente: lista de términos castellano-aymara.
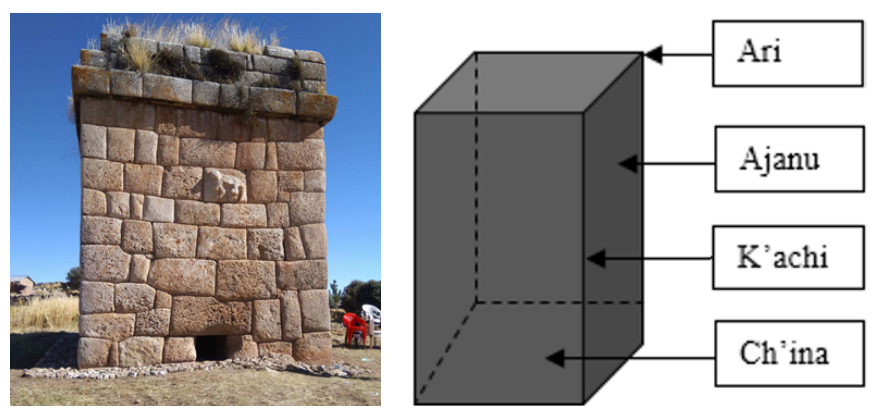

Figura 7. Chullpa cuadrada de Q'alaquta, Ilave (I). Elementos del sólido prisma (PIRWA) (D). Fuente: foto tomada por Henry Vilca-Apaza, 2019. (I), Recreación propia (D).

Prisma (pirwa).- Muchas chullpas presentan este cuerpo geométrico, poliedro regular cuyas caras laterales son paralelogramos y sus dos bases son polígonos, como las de Sillustani (Puno), Kutimbo (Puno), Sankuta (Pilcuyo), que en aymara es pirwa (según B). La chullpa de Q'alaquta, una comunidad ubicada a $8 \mathrm{Km}$ de la ciudad de Ilave, provincia de El Collao, departamento de Puno, presenta este cuerpo prismático de base cuadrangular, hecha de roca basáltica (ver Figura 7). Sus elementos se aprecian en la Tabla 10.

Finalmente, cabe agregar la rica terminología matemática y pedagógica acerca de la geométrica hallada en el estudio (ver Tabla 11).

\begin{tabular}{lll}
\hline \multirow{2}{*}{ Castellano } & \multicolumn{2}{c}{ Aymara } \\
\cline { 2 - 3 } Angosto & Equivalente & Glosa \\
\hline Arco & K'ullk'u & Ajustado \\
\hline Curva & Kürmi, k'umu & Arco iris, forma de arco \\
\hline Cuadriculado & Mujuna q'iwta, q'iwi, muyt'a & Dar vuelta a medias, regresa \\
\hline Cuerda & Kajunjata & Que está cuadriculado \\
\hline Diagonal & Wiska, phala, kira & Soga \\
\hline Diagrama (curva cerrada) & Q'ichusirqi, q'ichhu & Diagonal \\
\hline Espacio & Jullich'ipha, jist'atq'iwi & Figura cerrada \\
\hline Forma & Chiqa, pacha & El tiempo y espacio que vivimos \\
\hline Fila & Uñta & Como se ve \\
\hline Grado & Sipi, siqi & Ponerse uno tras otro \\
\hline & Q'iwi & Vuelta \\
\hline
\end{tabular}




\begin{tabular}{lll}
\hline Horizontal & Winkuchi, thaxsi & Que se echa o echado \\
\hline Línea & Sich'i, Sipi & Línea, raya \\
\hline Metro cuadrado & Payachata mitru & Metro al cuadrado \\
\hline Metro cúbico & Kimsachata mitru & Metro al cubo \\
\hline Oblicua & Q'ichu & Curvo \\
\hline Ondulada & Q'iwiq'iwi & Curvas y curvas \\
\hline Paralelepípedo & Sayt'utika, jikiwisajanu & Figura de lados iguales \\
\hline Paralelismo & Jikiwisäwi, payachisïwi & En el mismo sentido \\
\hline Rotación & Muyüwi & Que da vueltas \\
\hline Semiplano & Chikpallalla & La mitad de un espacio plano \\
\hline Simetría & Q'allüwi & Partido por la mitad \\
\hline Tamaño (qué) & Taña, tansa & Porte \\
\hline Traslación & Jithiyäwi & Que recorre \\
\hline Trazar & Rixsuña & Como rayar \\
\hline Vertical & Sayachi & Parado \\
\hline Zigzag & Link'u link'u & Que va y viene \\
\hline
\end{tabular}

Tabla 11. Términos aymaras anexos a la geometría.

Fuente: lista de términos castellano-aymara.

\section{CONCLUSIONES Y REFLEXIONES FINALES}

El aymara, al igual que otro pueblo, desarrolló una geometría que le sirvió de herramienta conceptual para organizar su pacha (tiempo-espacio), desarrollar tecnología, registrar sus vivencias y pensamientos simbólicamente en la textilería, agricultura, cerámica, arquitectura, etc., utilizando diversos símbolos geométricos y el lenguaje oral. Este área del saber encierra una rica terminología geométrica que además de concepto es un mensaje simbólico, por ejemplo, mujiña (triángulo) representa un sembrío en determinada época; pero también es evidencia del grado de abstracción conceptual y aplicación alcanzado en la geometría, acorde a su tiempo y contexto, no debiéndosele juzgar como inferior o superior.

El aymara, como lengua ágrafa, posee una vasta terminología especializada y propia sobre geometría y, ante la falta de ella, muestra un potencial léxico auxiliar que por aproximación conceptual puede ser adaptado al lenguaje matemático. Si bien desde la terminología geométrica castellana, el aymara muestra un vacío lexical matemático como en el caso de romboide, escaleno, metro, también muestra flexibilidad y capacidad morfosintáctica para adaptarse a las nuevas exigencias de la terminología científica y pedagógica y asimilar nuevos términos para vitalizarse a través de préstamos de lenguas primas como el quechua inclusive el castellano y creación de neologismos, al margen de la fidelidad lingüística. Esta terminología presentada y la que se genere ha de ser validada por especialistas 
Vilca, H., Sosa, G., (2020) Etnogeometría aymara: propuesta de terminología matemática para la escuela rural de Perú. Revista Latinoamericana de Etnomatemática, 13(2), 66-86

DOI:10.22267/relatem.20132.61

(lexicógrafos y filólogos) y por órganos pertinentes, cuidando la naturaleza lingüística del aymara.

Es preciso anotar que el aymara como lengua ha disminuido su acervo lexicológico matemático, debido a causas como la conquista quechua (Inka) y española, la política colonial de abandono de lenguas indígenas, la indiferencia producto de una educación alienante, la migración geográfica y cultural motivada por la búsqueda de mejores condiciones de vida en la ciudad, la negativa de los padres de enseñar a su hijos la lengua aymara por considerarla sinónimo de discriminación: Janiwa wawajaxa nayjamañapakiti ("No quiero que mi hijo sufra como yo"), es el clamor de los entrevistados. El estatus de la lengua castellana, al pertenecer al grupo de poder económico y político, es tal que ocasiona el sincretismo lingüístico en desmedro del aymara. La autenticidad sólo se preserva en las generaciones adultas, las relativamente jóvenes están adoptando términos castellanos a través de ese sincretismo que ellos llaman "aymañol" (aymara + español), es decir la aymaraización de términos castellanos como kajuna (cubo) que viene de "cajón", al que se agregó el sufijo locativo -na, válido socialmente pero cuestionado científicamente.

La importancia de la terminología sistematizada y propuesta no es cosa burda, es materia prima para los procesos de enseñanza aprendizaje en la escuela rural, al hacer más fluida la comunicación alumno-profesor, permitiendo optimizar el aprendizaje y cultivar la identidad y seguridad, tal cual es el propósito dualista de la educación matemática de D'Ambrosio, mejorar la calidad de vida y la dignidad, como indican Condori-Viza, Navarrete-Álvarez, Aguirre-Cipe \& Chamorro-Pérez (2017):

“...los estudiantes no sólo pudieron identificar diversos elementos de geometría tales como: transformaciones isométricas, ángulos, polígonos, teleselaciones, etc., sino que se trató de una experiencia educativa que generó procesos de identificación con el territorio y patrimonio arqueológico... sociales y culturales distintos" (Condori-Viza et al., 2017, p. 23).

Finalmente, reafirmamos que la enseñanza rural, en base a esta terminología, tiene un doble propósito: desde el ángulo cognitivo, mejorar el aprendizaje facilitando la comprensión y asimilación de conceptos ajenos a su cultura al relacionarlo con el suyo (partir de los 
saberes previos contextualizados propuestos por J. Piaget y L. Vygotski), y así nutrir su identidad y estima, desde el ámbito social, pues al ver valorada su cultura se sentirá valorado como humano, en la perspectiva de que la educación debe estar centrada en la condición humana (Morin,1999), y de la mano con la concepción aymara de que la "escuela debe elevarles a la condición de seres humanos para vivir con dignidad...de este modo, la complementariedad dialógica de sabidurías y conocimiento deben convertirse en el pilar de la construcción de una escuela rural" (Bermejo-Paredes et al., 2019, p.13).

\section{AGRADECIMIENTO}

Nuestra gratitud, a los participantes en la investigación de la comunidad de Sacari Titikachi del distrito de Pilcuyo y de Moho, departamento de Puno, Perú; a nuestro guía, al Sr. Miguel Mamani (de quien aprendimos que "Mamani” significa halcón en lengua aymara); a la Sra Francisca Apaza por la logística brindada y por inspirarnos futuras investigaciones.

\section{REFERENCIAS BIBLIOGRÁFICAS}

Arias M., P.P. (2005). Etnomatemática en la escuela primaria. Puno: Editorial Titikaka.

Ascher, M. \& Ascher, R. (1986). Etnomatemática. Granada: Sin editorial.

Bermejo-Paredes, S., \& Maquera-Maquera, Y. (2019). Interpretación de la escuela rural andina en comunidades aimaras de Puno-Perú. Revista Electrónica Educare, 23(2), 1-15. Recuperado de https://doi.org/10.15359/ree.23-2.4

Bertonio, L. ([1612] 1993). Transcripción del vocabulario de la lengua aymara. La Paz: Edición facsimilar de Radio San Gabriel - Instituto Radiofónico de Promoción Aymara IRPA.

Cerrón-Palomino, R. (2000). Lingüistica aimara. Cuzco: Centro de Estudios Regionales Andinos "Bartolomé de las Casas".

Condori-Viza, C., Navarrete-Álvarez, M., Aguirre-Cipe, I., \& Chamorro-Pérez, A. (2017). Cultura Arica: Un caso para el estudio y educación de la geometría presente en textiles prehispánicos. Revista Latinoamericana de Etnomatemática, 10(2), 8-25.

D’Ambrosio, U. (2005). Sociedade, cultura, matemática e seu ensino. Educação e Pesquisa. Revista da Faculdade Educação da USP, 9(1), 99-120.

D’Ambrosio, U. (2008). O Programa Etnomatemática: uma síntese. Rev. Acta Scientiae, 10(1), 716.

De la Hoz M., E., Trujillo Varilla, O., \&Tun, M. (2017). La Geometría en la Arquitectura de la vivienda tradicional Arhuaca. Revista Latinoamericana de Etnomatemática. 10 (1), 1-14.

Deza G., J.F. (1989). Jaya mara aru: nuevo diccionario aymara-castellano, castellano-aymara. Lima: Graphos 100 editores. 
Vilca, H., Sosa, G., (2020) Etnogeometría aymara: propuesta de terminología matemática para la escuela rural de Perú. Revista Latinoamericana de Etnomatemática, 13(2), 66-86

DOI:10.22267/relatem.20132.61

Fernández, L. (abril 2, 2008). Esta es la joya más antigua de Puno. En El Gran Sur, La República. Lima, Perú: Imp. Talleres del Grupo La República.

Ferreira, J. C., \& Neves-Rogério, M. (2017). JOIAS DO ASÉ: Sobrevivência, transcendência e etnogeometria relacionados à sua produção na comunidade Casa do Boneco de Itacaré. Revista Latinoamericana de Etnomatemática, 10(3), 59-77.

Gonzáles H., D. ([1608] 1989). Vocabulario de la Lengua General de todo el Perú llamada Lengua Qquichua o del Inca. $3^{\text {a }}$ edición. Lima: Edición facsimilar de Editorial, Imprenta, Biblioteca Central y Librería de la Universidad Nacional Mayor de San Marcos.

Guerra, S. \& Guerra, J. (2017). Fundamentos epistémicos complejos de la educación intercultural bilingüe. Revista Educación y Humanismo, 19(33), 441-455. Recuperado de http://dx.doi.org/10.17081/eduhum.19.33.2655

Kesseli, R. \& Pärssinen, M. (2005). Identidad étnica y muerte: torres funerarias (chullpas) como símbolos de poder étnico en el altiplano boliviano de Pakasa (1250-1600 d. C.). Bulletin de l'Institut français d'études andines, 34 (3). Recuperado de http://journals.openedition.org/bifea/4936; DOI: https://doi.org/10.4000/bifea.4936

Layme P., F. (2004). Diccionario bilingüe aymara castellano. Bolivia: Consejo Educativo aymara.

López, L.E. (1988). La escuela en Puno y el problema de la lengua: excurso histórico (1900-1970). En L.E. López, (Edit.), Pesquisas en lingüistica andina (pp. 256-332). Lima, Perú: Gráfica BELLIDO.

Ministerio de Educación. (1905). Vocabulario políglota incaico. Lima: Tipografía del Colegio de Propaganda FIDE del Perú.

Ministerio de Educación. (1985, noviembre 18). Resolución Ministerial No $1218-85$-ED Alfabeto quechua y aimara. Recuperado de http://www.drelp.gob.pe/DIGEIBIRA/COMUNICADOS/RM\%201218-85ED\%20QUECHUA\%20y\%20AIMARA.pdf

Ministerio de Educación. (2002). Jakhuri Masija. Lima: Quebecor World Perú S.A.

Morín, E. (1999). Los siete saberes necesarios para la educación del futuro. (Traducción de Mercedes Vallejo-Gómez). París: UNESCO. Recuperado de http://www.ideassonline.org/public/pdf/LosSieteSaberesNecesariosParaLaEdudelFuturo.pdf

Palao B., J. B. (2005). Etnohistoria del Altiplano de Puno. Puno: Arte y Color E.I.R.L.

Reynoso, C. (2019). Etnogeometrías: Patrones geométricos y cultura. Recuperado de http://carlosreynoso.com.ar/archivos/etnogeometria/Carlos-Reynoso-Etnogeometria.pdf

Vilca Apaza, H. M., Yapuchura Saico, C. R., Mamani Apaza, W. W, \& Sardón Ari, D. L. (2018). Maestros indigenistas y sus experiencias socio-educativas en el altiplano peruano en el siglo XX.Comuni@cción,9(2), 90-100. Recuperado de http://www.scielo.org.pe/scielo.php?script=sci_arttext\&pid=S2219$71682018000200002 \& \operatorname{lng}=$ es\&tlng=es. 